\title{
Correction to: Fused features mining for depth-based hand gesture recognition to classify blind human communication
}

\author{
Saba Joudaki ${ }^{1}$ - Dzulkifli Mohamad ${ }^{2} \cdot$ Tanzila Saba $^{3}$ - Abdulaziz S. Almazyad ${ }^{4,5}$ • \\ Amjad Rehman ${ }^{5}$
}

Published online: 2 December 2017

(C) The Natural Computing Applications Forum 2017

Correction to: Neural Comput \& Applic (2017) 28:3285-3294

https://doi.org/10.1007/s00521-016-2244-5

In the original publication, the first author name and his affiliation were incorrectly published. The correct author name and his affiliation are as follows:

\section{Saba Joudaki}

Assistant Professor, Department of Computer Engineering, Technical and Engineering Faculty, Islamic Azad University, Khorramabad, Iran.

The original article can be found online at https:// doi.org/10.1007/s00521-016-2244-5.

Amjad Rehman

a_khan@yu.edu.sa

1 Department of Computer Engineering, Technical and Engineering Faculty, Islamic Azad University, Khorramabad, Iran

2 Faculty of Computing, Universiti Teknologi Malaysia, 81310 Skudai, Johor, Malaysia

3 College of Computer and Information Sciences, Prince Sultan University, Riyadh, Kingdom of Saudi Arabia

4 College of Computer and Information Sciences, King Saud University, Riyadh, Kingdom of Saudi Arabia

5 College of Computer and Information Systems, Al-Yamamah University, Riyadh, Kingdom of Saudi Arabia 